International Journal of Engineering \& Technology, $7(2.23)(2018) 483-488$
International Journal of Engineering \& Technology
SPC
Website: www.sciencepubco.com/index.php/IJET
Research paper

\title{
Short Review of Studies on Modeling of Technology and Technical Means Used for Production of Classical and Nanomodified Functional Polymer Composite Materials
}

\author{
A. E. Kolosov ${ }^{1}$, E. P. Kolosova ${ }^{1}$ \\ ${ }^{I}$ National Technical University Of Ukraine «Igor Sikorsky Kyiv Polytechnic Institute», \\ 37 Prospect Peremohy, Kyiv, 03056, Ukraine \\ *Corresponding Author E-Mail: A-Kolosov@Ukr.Net
}

\begin{abstract}
The paper briefly announces the results of many years of research in the field of modeling and design of parameters of technology and technical means for production of classical and nanomodified functional polymer composite materials for structural purposes. Models of oriented structurally inhomogeneous media are considered which form the basis for designing parameters for technological methods of impregnation and winding, as well as for predicting the stress-strain state of classical polymer composite materials. Ultrasonic treatment is being investigated as a basic method of intensifying technological processes as well as improving the physico-mechanical and operational properties of classical and nanomodified functional polymer composites. The main parameters and features of the ultrasonic treatment modes are described. The results of modeling and designing of manufacturing technology parameters for production thermoplastics are presented. The aspects of the production technology for nanomodified functional polymer composite materials for functional purposes are provided.
\end{abstract}

Keywords: Modeling; Design; Technology; Composite; Nanomodification; Ultrasonic.

\section{Introduction}

Constructional polymer composite materials (PCM) for functional purposes are now widely used in various industries. In particular, it is chemical and polymer engineering, aviation, rocket and shipbuilding, automotive, construction, medicine, electrical engineering, utilities, sports and other industries. And every year the range and scope of such materials is expanding.

The requirements imposed on composite materials used in structural components of structures are primarily determined by their functional purpose. Among the basic requirements - high strength and rigidity, resistance to alternating loads, low mass, high long-term strength, thermal and corrosion resistance, tracking resistance, and high reliability. Although other requirements may be added to the above basic requirements for the specific composite material, the conditions of its operation.

For example, it is known that nanomodified (NM) PCMs (NMPCMs), which contains fibrous fillers (for example, carbon fibers, carbon nanotubes - CNTs) and reactoplastic polymer binders (PBs), for example, epoxy binders (EBs) are primarily intended for use in highly loaded and highly critical power products.

To enhance the effect, such PBs in the liquid state are modified by various nanoparticles, mainly carbon-based (fullerenes, astralenes, CNTs, graphenes, graphene aerogels, etc.).

A wide range of methods are widely used to predict the design and technological parameters of technical equipment intended for the production of classical PCMs and NM PCMs for functional purposes. Among them are methods of mathematical, structuralparametric and computer modeling. Some studies, in which the most significant results are obtained on the investigated problem, are briefly described below.

\section{Modelling the Parameters of the Structure and Technologies for Obtaining Classical React plastic Polymer Composite Materials}

\subsection{Structural Models of Oriented Structural-Non- Homogeneous Media}

The starting point of the structural-parametric modelling of oriented fibrous composites is the elaboration of adequate geometric models for such structures. So, for example, models of structurally inhomogeneous media [1], applied to classical fibrous reactive plastic PCMs, allow solving at least two problems simultaneously.

First, they allow you to design the parameters of the main technological operations of the process of obtaining classical oriented PCMs (impregnation, winding, dosing, drying, etc.). Secondly, knowing the geometric parameters of the adequate structural models, as well as the physical and mechanical properties of the reinforcing filler and the polymer matrix, it becomes possible to predict the stress-strain state of the composites produced.

This makes it possible to control the functional applicability of a composite obtained using a specific technology. Therefore, it is important to determine the parameters of such structural models. 
Some developed approaches to constructing adequate models of structurally inhomogeneous media and predicting their parameters are described in $[2,3]$.

\section{Ultrasound as the Dominant Method for Production of Classical and Nanomodified Polymer Composite Materials}

\subsection{Production of Classical Polymer Composite Materials Using Ultrasonic}

Despite the rather long history of creating ultrasonic (US) technology and its application in various fields, such processing is currently the dominant method of physical modification Moreover, low-frequency US cavitation plays a special role in the production (or synthesis) for both classical PCMs and NM PCMs. Among other positive effects, US processing increases the productivity of the process of obtaining classical PCMs. Also, in the optimum, the cumulative hardening time of the sonicated reactoplasts is reduced.

When the liquid media is sonicated at high intensities, the cycles of high and low pressures are successively alternated. This alternation occurs at speeds that depend primarily on the frequency of US. During the second phase (rarefaction), small bubbles or cavities are formed in the liquid in which steam or gas is located. After the emerging bubbles reach a volume at which they can no longer absorb acoustic energy, they burst (implosion) during the onset of the high-pressure cycle (compression). This causes US cavitation.

A theoretical estimate of the values of the parameters locally attained during implosion is characterized by temperature (about $5000 \mathrm{~K}$ ), pressure (about $200 \mathrm{MPa}$ ), heating and cooling rates of $>10^{10} \mathrm{~K} / \mathrm{s}$, as well as the rate of outflow of liquid jets up to 280 $\mathrm{m} / \mathrm{s}$. Therefore, it is important to develop processes and production capacities for cavitation processing. As a result, US vibrations are generated, which are required for specific technological processes (operations).

For PCMs, the main technological parameters of US processing are frequency, intensity, amplitude, time, temperature and pressure. Some problem situations for the main production cycle in the preparation of reinforced PCMs based on epoxy polymers (EPs) are considered in study [7]. The feasibility investigations of the use of US for the physical modification of EPs have been substantiated by the studies carried out.

As a result of such modification with optimal processing parameters, energy savings are achieved, and the quality and performance characteristics of the sonicated PCMs are improved. Known effective methods for processing of liquid reactoplastic media using low-frequency US modification are analyzed in [8]. Epoxy oligomers (EOs) and epoxy compositions (ECs) based on them were investigated as liquid reactoplasts. As a result of effective US treatment regimes, the technological and operational properties of hardened composites have been improved.

An emergence of US cavitation in liquid media depends on the variation in the parameters of US treatment. Among such parameters an important place is occupied by frequency, pressure, time and temperature. Moreover, the optimal combination of effective processing parameters should be determined purely by an experimental method with subsequent reflection of the results obtained in the form of experimental-statistical models.

The effect of low-frequency processing of US treatment of reactoplastic PCMs (not filled and filled with short-fiber fillers) with a variation of excess pressure was experimentally investigated in [9]. The results of the investigations made it possible to determine the optimal parameters of the US treatment of liquid EC.

The relationship between low-frequency US treatment and the operational properties of solidified PCMs is discussed in the study [10]. Comparison of the efficiency of the developed US technology in comparison with the existing methods of obtaining classical PCMs is made.

The rationale for the effectiveness of the variation of the frequency US range for the sonication of liquid $\mathrm{EC}$ is given in [11] The end result of this treatment was an increase in the operational properties of solidified EPs. The results of the experimental studies made it possible to establish a set of effective parameters of US treatment. Also, there was a decrease in energy costs by 2 2.5 times when obtaining EPs at effective regimes.

\subsection{Intensification of the Impregnation Process and Strengthening of Composites}

Proceeding from the above, it follows that the basic prerequisites for the use of US are based on the physical and chemical effects that arise when pulsating bubbles of vapor and gas with cavitation action.

Intensification of production of reactoplastic PCMs during US cavitation is analyzed in $[12,13]$. The relationship between the effective parameters of US treatment with the operational properties of EBs and classical woven fibrous PCMs on their basis is considered in [14].

The results of experimental tests of ring winding samples of classical PCMs from unidirectional fillers in the form of glass fibers and organic fibers using low-frequency US vibrations are given in [15].

On the basis of the study of the strength characteristics of ring samples, optimal modes and parameters of US treatment were established, among them: frequency, amplitude, intensity and time. Optimization was carried out on the basis of the study of the relationship between the values of tensile strength, compression, bending and interlayer, as well as the relative content of filler, depending on processing parameters.

As a result of effective US treatment, the defectiveness of the composites was reduced as a result of a reduction in the content of air inclusions, as well as an improvement in the distribution of the PB in the interfiber space.

It has been experimentally established that the deviation of the effective parameters of US treatment from optimal values leads to a decrease in the performance characteristics of solidified oriented PCMs.

\section{Designing of Technical Tools Parameters for Obtaining of Classical Polymer Composite Materials Using Ultrasonic}

A block of researches related to the development of approaches to the design of constructional and technological parameters of technical means for US processing in the production of classical PCMs is presented in papers [16 - 18]. Technical means of US processing include the development of appropriate devices and technological methods.

A developed approach to the selection of the regime parameters of the method, as well as the effective design parameters of US technological equipment, which implements the developed method is described in [16]. It is indicated that the use of the developed method provides an increase in the productivity of US treatment of approximately 2-2.5 times. This, in turn, makes it possible to intensify the processes of 'free' impregnation, dosing of impregnated fibrous fillers and their wet winding on the mandrel when forming classical winding PCMs.

An analytical technique for calculating a composite dozing device based on piezoceramic transducers connected to a rectangular radiating plate that undergoes bending vibrations along and across the working surface is presented in [17]. As an example, calculations are made of the effective acoustic dimensions of the composite elements of a sectional piezoelectric transducer, depending on the frequency of US vibrations and the material used to fabricate the elements. The dosing device, manufactured on the basis of the developed technique, was aphrodised in the process of 
contact treatment of a glass fiber cloth with a width of $1120 \mathrm{~mm}$ impregnated with an EB.

On the basis of complex studies, a conceptual approach to the application of the principles of computer-aided design was proposed in [18]. The developed approach was adapted to determine the consrtuctional-technological parameters of technical means for molding plastic fiber PCMs with the use of lowfrequency US treatment in cavitation mode. The approach was based on the methodology of structural-parametric geometric modelling of complex objects and systems.

As part of the adaptation of the developed approach, a general structural and parametric model of the technological process for the preparation of classical fibrous PCMs was proposed. According to the developed approach, this model was presented in the form of three consolidated blocks. The first of which is a block of US processing of epoxy resin in and preparation of impregnating ECs. The second block is a block of free impregnation of the fibrous material with ECs in an impregnating bath. The third block is a unit for adjusting the content of PBs in impregnated fibrous material (block of dosed deposition). The proposed approach is advisable to apply to the entire production cycle of oriented fibrous PCMs using US treatment.

\section{Modelling Parameters of Technology and Technical Means for Thermoplastics Molding}

\subsection{Manufacturing Technology of Thermoplastics: Modelling and Designing}

The development of approaches to the modelling of constructive and technological means for the production of thermoplastics, unlike thermosets, has its own features and differences. First of all, this distinction is due to a significant difference in the rheological characteristics of the processed polymers (in particular, their dynamic viscosity), as well as processing conditions (temperature, pressure). Therefore, for thermoplastics, it is necessary to use other approaches.

Thermoplastics are represented by a wide range of manufactured products, in particular profile, blown, and tubular products [1923]. Features of the implementation of the production process, including the design features of the process equipment for the molding of corrugated tubular products based on thermoplastics, are described in [19]. In particular, the effects of volume changes, as well as the effect of subsequent stages of the production cycle, were studied when forming a tube piece blank by the wall thickness of the resulting pipe billet. As an optimizing factor, the productivity of forming equipment is accepted.

In the development of the results of the study [19], an improved analytical approach to determine the basic geometric parameters of corrugated tubular products from thermoplastics is proposed in study [20]. The proposed approach allows to establish the dependence of the structurally specified parameters of the corrugated products, the chosen corrugating scheme with the geometric dimensions of the profile of the corrugated tubular products. Within the framework of the developed approach, three variational molding schemes are considered. Among them - free shaping corrugation arch, forming high-strength corrugations with inclined walls, as well as forming low-profile corrugations.

Simulation of one of the stages of the technological process of forming a polymeric corrugated tubular product, namely the process of welding several layers of a pipe, is given in [21]. Mathematically formulated the necessary conditions for welding of polymer layers, as well as a numerical solution of the temperature problem in the welding zone.

The project profiling of corrugated pipes of different geometries on the basis of the developed numerical-experimental technique is carried out in [22]. Variants of forming corrugations of various geometries are presented. In particular, low-profile corrugations, as well as high-strength corrugations with vertical and inclined walls with a flat and circular arc.
The approaches developed in studies [19 - 22] were applied to modelling the process of extrusion blow molding of a polymer billet. In particular, it was found that this process is characterized by two different stages [23]. Analytical dependencies have been obtained that determine the relationship between the design and process parameters of the extrusion blow molding process with the geometric parameters of the blown product.

The block of studies [24 -26] concerns modelling of constructive and technological parameters of technical means for molding polymeric products from thermoplastic by extrusion methods. This includes extruder extruders, extrusion heads and the process of mixing in an extruder.

The process of non-isothermal mixing in a cylindrical mixer of an extruder based on numerical simulation was studied in [24]. An analytical distribution of the transfer rates of the polymer melt, which flows in the effective channels of the drum mixer, was obtained. Simulations were carried out at various temperatures and strains. This also took into account the design of the mixer.

Modelling of the flow of a viscoelastic fluid flow at the exit from the working tool of an extruder (extrusion head) was carried out in [25] on the basis of the mathematical model Phan-Thien-Tanner. The results of numerical simulation can be used to select effective modes of extrusion processing, as well as to design advanced designs of extrusion heads.

The developed improved physical and mathematical models of the melting process of a thermoplastic polymer in screw channels of a worm extruder are described in [26]. According to the proposed models, three phases interact in the melting zone at once. The first phase is a solid porous cork. The second phase consists of a mixture of melt, the remains of solid granules and gas. The third phase is represented a completely melted thermoplastic material. Moreover, the velocity and temperature distributions of the material in the auger channels are obtained taking into account the influence of complex factors. Among the latter are the processes of barodiffusion of the convection, as well as the collapse of the plug.

The last study [26] in the block of thermoplastics concerns the implementation of one of the structural and technological approaches to the thermal modernization of existing residential buildings and structures. In particular, a solution is offered that provides thermal insulation of the external facade and the use of heat-insulating elements based on PCMs. The latter can also be obtained using US treatment.

\subsection{Connection and Repair of Polyethylene Pipelines Using Ultrasonic Modification and Heat Shrinkage}

The block of studies [28 - 32] describes the features of the developed technical means that relate to effective hardware for connection and repair of polyethylene (PE) pipelines using US modification and heat shrinkage.

The article [28] deals with the connection and restoration of polymer pipelines based on PE. The connection of damaged sections of pipelines intended for the transportation of natural gas is carried out on the basis of melt-glue technologies using US treatment. ECs are used as glue compositions, and heat shrink couplings (muffs) are formed on the basis of EPs. It has been experimentally established that an effective low-frequency US treatment leads to an increase in the performance characteristics of the molded adhesive-glued compounds. In particular, their deformation and adhesion strength. In addition, there is a decrease in the level of residual stresses in the repaired pipelines, which allows to extend the period of their operation.

The paper [29] describes an improved methodology for forming epoxy repair couplings with shape memory effect based on the use of effective US treatment regimes. At the same time, the maximum deformation of the molded tubular blanks from EPs formed without US treatment was $15-17 \%$ at a diameter of $63 \mathrm{~mm}$. Effective regime parameters of US treatment based on studying the correlation of the maximum deformation hardening of tubular 
blanks from EPs and the permissible degree of their deformation are established.

The paper [30] describes the developed complex methods of connecting damaged PE pipes in the field conditions. These methods combine preliminary surface treatment of PE pipes to increase their surface energy. Further, the ends of the PE pipes are bandaged together with repair couplings and a model glass fiber which is preliminary impregnated with EC. It is established that the practical implementation of the developed complex technology results in a 55-60\% increase in the performance of the repair connection.

Features of implementing another technology for connecting and repairing damaged sections of PE pipelines, as well as its advantages over traditional technologies, are described in study [31]. The authors proposed the use of epoxy-adhesive compounds and various variants of bandaging damaged PE pipelines with the use of effective US exposure regimes. In this case, as an option, contact surface activation of the surface of the connected sections of PE pipes by a concentrator of US vibrations is provided.

Features of the use of thermistor couplings for repair in the field conditions of low- and medium-pressure PE gas pipelines are considered in [32]. Effective parameters of the thermal resistive welding process were established. In particular, the limiting value of electrical resistance, welding time, welding voltage, as well as the time required for cooling the butt joints of the end sections of PE pipelines. Effective parameters of the process of thermal resist welding were established depending on the diameter of the PE pipelines to be connected, the standard dimensions and the materials for the manufacture of connecting thermistor couplings, as well as the conditions for the process.

\section{Aspects of Production Technology for Nanomodified Polymer Composites Functional Purpose}

Nanotechnology is the scientific and technological priority chosen by the governments of advanced countries, for which they annually allocate billions in national currencies. Nanomaterials with exceptional performance properties directly cross the line between nanoscience and nanotechnology. At the same time, the role of carbon nanostructures, dominants among all nanofillers, is leading at the stage of evolutionary nanotechnology. The goal of evolutionary nanotechnology is not simply the obtaining of an improving effect as such, but the construction (modelling, synthesis) of effective nanomaterials and nanodevices with predefined functions and operational properties. This allows to consider carbon nanofillers (primarily fullerenes, CNTs, graphenes and aerogels of graphene) as a convenient "model" for discussing and approbating a number of methods that can help in solving actual program problems.

Prospective directions of development and potential application of nanotechnologies, including for the production of constructional NM PCMs for functional applications, taking into account the success of modern science and technology in the field of polymer nanomaterial science, are discussed in [33]. Prospective directions of development of NMPCMi production technology are analyzed taking into account the economic aspects of their implementation and possible branches of applications.

A number of effective patent-protected technical devices (methods and devices) of the Russian Federation using low-frequency US cavitation is analyzed in [34]. Particular attention was paid to the technical means used for the production of reactoplastic NMPCMs. It was noted that the use of the developed technical means contributes to the increase in strength and lifetime of structures based on NMPCMs. Also, the main problem situation was noted, related to the difficulty of ensuring a uniform distribution of nanoparticles in the liquid oligomers. The latter is due to the physico-chemical nature of carbon nanofillers and their mutual attraction. This facilitates the aggregation of nanoparticles to each other and the formation of agglomerates in a liquid oligomer.
The effective methods for dispersing carbon nanofillers in liquid media using the example of CNTs are described in [35]. As liquid media, organic solvents and liquid polymer media were used. The relationship between effective dispersing methods to improve the performance characteristics of the final NMPCMs produced from them was studied. It was shown that the necessary conditions for the creation of such materials with improved operational properties are connected with small dimensions and the most uniform distribution of carbon nanoparticles in the bulk of the liquid polymer matrix.

Peculiarities of obtaining reactoplastic NMPCMs using modification of EO with CNT, and some problematic situations associated with this process, were considered in the article [36]. The results of obtaining a dispersion of highly viscous suspensions based on EO with CNT by the dispersion method in a three-roll mill in comparison with the sonication of dispersions in the regime of low-frequency US cavitation were analyzed. It was shown that the effectiveness of the introduction of CNTs in a liquid polymer medium depended not only on the administered dose of CNT, but also on the regime parameters of US mixing.

Retrospective aspects of obtaining carbon plastics and the characteristics of reactoplastic NMPCMs produced in the Russian Federation on the basis of carbon fiber prepregs were considered in [37]. The efficiency of the use of structural carbon plastics in the bearing elements of structures of highly loaded elements of aircraft under conditions of alternating loads is shown. The improvement of the physical and mechanical properties of the final product by creating a combined filling of carbon-fiber-plastic is experimentally proved. In this connection, a continuous carbon fiber is impregnated with a PB in which the ultradispersed carbon nanoparticles were pre-distributed evenly.

It was stated above that the extraordinary conditions arising from the US action of cavitation regimes can improve existing materials, and also synthesize a wide range of promising nanostructured materials [38]. For example, US cavitation processing is an effective method for the production of high-quality graphene, and in large quantities [39]. For example, graphite is added to the mixture of dilute organic acid, alcohol and water. Then the obtained mixture is subjected to US treatment at effective regimes [40].

A wide range of issues concerning with production and properties of functional materials for construction applications based on classical and nano composites are investigated in [41]. And the main attention is paid to US treatment as the dominant method of physical modification in the production of PCMs for functional purpose with predetermined operational properties.

\section{Conclusion}

The analysis of the above mentioned studies, in which various aspects of forecasting the design and technological parameters of technical equipment intended for the production of classical PCMs and NM PCMs for functional purposes are considered, allows us to draw the following conclusions.

Determination of constructive-technological means for forming of classical PCMs and NM PCMs is expedient to implement on the basis of complex use of various configuration of modelling methods. In particular, by using mathematical, physical, and also experimental-statistical models.

At the present stage of studying and synthesizing both classical and nanomodified PCMs, the physical and chemical modification of the surface of reinforcing fibers and liquid PBs is the main direction in the development of functional design-oriented PCMs. The effectiveness of physical modification in the form of lowfrequency US vibrations in the production of functional classical PCMs and NM PCMs is ensured by a whole complex of structural and operational indicators. Among them - an increase in structural homogeneity, intensification of impregnation, improvement of technological properties of the sonicated liquid polymer matrix. 
No less important is the increase in the physico-mechanical and operational properties of the solidified polymer matrix.

The use of low-frequency US is an effective method of aggregate disintegration of nanofillers, including in the production process of NMPCMs.

Increasing the effectiveness of US treatment is determined by a set of effective interrelated parameters of US processing, which are determined experimentally for a particular technological process. In particular, the frequency, intensity, amplitude, temperature and pressure.

The obtained results provide directions for further research on energy and resource efficiency in the production of constructional classical PCMs and NM PCMs for functional purposes.

\section{References}

[1] Kichigin AF, Kolosov AE, Klyavlin VV \& Sidyachenko VG (1988), Probabilistic-geometric model of structurally inhomogeneous materials. Soviet Mining Sci. 24, I. 2, 8794.http://dx.doi.org/10.1007/bf02497828.

[2] Kolosov AE \& Klyavlin VV (1988), Determination of the parameters of a geometric model of the structure of directionally reinforced fiber composites. Mech. Compos. Mater. 23, I. 6, 699706. http://dx.doi.org/10.1007/bf00616790.

[3] Kolosov AE \& Klyavlin VV (1989), Several aspects of determination of the adequate model of the structure of oriented fiber-reinforced composites. Mech. Compos. Mater. 24, I. 6, 751757. http://dx.doi.org/10.1007/bf00610779.

[4] Kolosov AE (1988), Impregnation of fibrous fillers with polymer binders. 1. Kinetic equations of longitudinal and transverse impregnation. Mech. Compos. Mater. 23, I. 5, 625633.http://dx.doi.org/10.1007/bf00605688.

[5] Kolosov AE, Repelis IA, Khozin VG, Klyavlin VV (1988), Impregnation of fibrous fillers with polymer binders. 2. Effect of the impregnation regimes on the strength of the impregnated fillers. Mech. Compos. Mater. 24, I. 3, 373380.http://dx.doi.org/10.1007/bf00606611.

[6] Kolosov AE \& Repelis IA (1989), Saturation of fibrous fillers with polymer binders 5 . Optimization of parameters of the winding conditions. Mech. Compos. Mater. 25, I. 3, 407415.http://dx.doi.org/10.1007/bf00614811.

[7] Kolosov AE, Sakharov AS, Sivetskii VI, Sidorov DE \& Sokolskii AL (2012), Substantiation of the efficiency of using ultrasonic modification as a basis of a production cycle for preparing reinforced objects of epoxy polymer composition. Chem. and Petrol. Eng. 48, I. 5-6, 391-397.http://dx.doi.org/10.1007/s10556012-9629-9.

[8] Kolosov AE (2014), Low-Frequency Ultrasonic Treatment as an Effective Method for Modifying Liquid Reactoplastic Media. Chem. and Petrol. Eng. 50, I. 1-2, 7983.http://dx.doi.org/10.1007/s10556-014-9859-0.

[9] Kolosov AE (2014), Low-Frequency Ultrasonic Treatment of Liquid Reactoplastic Media with Pressure Variation. Chem. and Petrol. Eng. 50, I. 5-6, 339-342.http://dx.doi.org/10.1007/s10556014-9904-z.

[10] Kolosov AE (2014), Effect of low-frequency ultrasonic treatment regimes on reactoplastic polymer composite material operating properties. Chem. and Petrol. Eng. 50, I. 3-4, 150 155.http://dx.doi.org/10.1007/s10556-014-9871-4.

[11] Kolosov AE (2014), Efficiency of liquid reactoplastic composite heterofrequency ultrasonic treatment. Chem. and Petrol. Eng. 50, I. 3-4, 268-272. http://dx.doi.org/10.1007/s10556-014-9893-y.

[12] Kolosov AE, Karimov AA, Khozin VG, Klyavlin VV (1989), Impregnation of fibrous fillers with polymer binders. 3. Ultrasonic intensification of impregnation. Mech. Compos. Mater. 24, I. 4, 494-502. http://dx.doi.org/10.1007/bf00608132.

[13] Kolosov AE (2014), Prerequisites for using ultrasonic treatment for intensifying production of polymer composite materials. Chem. and Petrol. Eng. 50, I. 1-2, 11-17. http://dx.doi.org/10.1007/s10556014-9846-5.

[14] Karimov AA, Kolosov AE, Khozin VG \& Klyavlin VV (1989), Impregnation of fibrous fillers with polymer binders. 4 Effect of the parameters of ultrasound treatment on the strength characteristics of epoxy binders. Mech. Compos. Mater. 25, I. 1, 82-88. http://dx.doi.org/10.1007/bf00608456.

[15] Kolosov AE, Karimov AA, Repelis IA, Khozin VG \& Klyavlin VV (1990), Impregnation of fibrous fillers with polymeric binders.
6. Effect of parameters of ultrasound treatment on strength properties of wound fibrous composites. Mech. Compos. Mater. 25, I. 4, 548-555. http://dx.doi.org/10.1007/bf00610711.

[16] Kolosov AE, Sakharov AS, Sivetskii VI, Sidorov DE \& Sokolskii AL (2012), Method of selecting efficient design and operating parameters for equipment used for the ultrasonic modification of liquid-polymer composites and fibrous fillers. Chem. and Petrol. Eng. 48, I. 7-8, 459-466. http://dx.doi.org/10.1007/s10556-0129640-1.

[17] Kolosov AE, Sivetskii VI, Kolosova EP \& Lugovskaya EA (2013), Procedure for analysis of ultrasonic cavitator with radiative plate. Chem. and Petrol. Eng. 48, I. 11-12, 662672.http://dx.doi.org/10.1007/s10556-013-9677-9.

[18] Kolosov AE, Virchenko GA, Kolosova EP \& Virchenko GI (2015), Structural and technological design of ways for preparing reactoplastic composite fiber materials based on structural parametric modeling. Chem. and Petrol. Eng. 51, I. 7-8, 493-500. http://dx.doi.org/10.1007/s10556-015-0075-3.

[19] Kolosov AE, Sakharov AS, Sidorov DE, Sivetskii VI (2012), Aspects of profile shaping of corrugated tubular components. Part 3 Extrusion shaping of tubular polymeric blanks for manufacture of corrugated pipes. Chem. and Petrol. Eng. 48, I. 3-4, 199-206. http://dx.doi.org/10.1007/s10556-012-9598-z.

[20] Kolosov AE, Sakharov AS, Sidorov DE \& Sivetskii VI (2012), Aspects of profile shaping of corrugated tubular components. Part 1 Modeling of parameters of different profiles of corrugations, and also their shaping equipment. Chem. and Petrol. Eng. 48, I. 1-2, 60-67. http://dx.doi.org/10.1007/s10556-012-9575-6.

[21] Kolosov AE, Sakharov AS, Sidorov DE \& Sivetskii VI (2012), Manufacturing Technology: Aspects of profile shaping of corrugated tubular components. Part 2. Modeling the extrusion welding of layers of corrugated tubular articles. Chem. and Petrol. Eng. 48, I. 1-2, 131-138. http://dx.doi.org/10.1007/s10556-0129588-1.

[22] Sidorov DE, Sivetskii VI, Kolosov AE \& Sakharov AS (2012), Shaping of corrugation profiles during production of corrugated tubular articles. Chem. and Petrol. Eng. 48, I. 5-6 384 390. http://dx.doi.org/10.1007/s10556-012-9628-x.

[23] Sidorov DE, Kolosova EP, Kolosov AE \& Shabliy TA (2018), Analysis of blown process for producing polymer products by extrusion blow molding. East.-Eur. Journ. of Enterp. Technol. 2/1 (92) 14-21. doi: 10.15587/1729-4061.2018.126015

[24] Sakharov AS, Kolosov AE, Sokolskii AL \& Sivetskii VI (2012), Modeling the mixing of polymeric composites in an extrusion drum mixer. Chem. And Petrol. Eng. 47, I. 11-12, 799805.http://dx.doi.org/10.1007/s10556-012-9553-z.

[25] Kovalenko KG, Kolosov AE, Sivetskii VI \& Sokolskii AL (2014), Modeling Polymer Melt Flow at the Outlet from an Extruder Molding Tool. Chem. and Petrol. Eng. 49, I. 11, 792-797. http://dx.doi.org/10.1007/s10556-014-9837-6.

[26] Sakharov AS, Kolosov AE, Sivetskii VI \& Sokolskii AL (2013), Modeling of Polymer Melting Processes in Screw Extruder Channels. Chem. and Petrol. Eng. 49, I. 5-6, 357-363. http://dx.doi.org/10.1007/s10556-013-9755-z.

[27] Yeromin AV, Kolosov AE (2018), Modeling of energy effective solutions regarding the heating system and facade heat insulation during implementation of thermomodernization. East.-Eur. Journ. of Enterp. Technol. 1/8 (91) 49-58. doi: 10.15587/17294061.2018.123021

[28] Kolosov AE, Sakharov OS, Sivetskii VI, Sidorov DE \& Pristailov SO (2011), Effective hardware for connection and repair of polyethylene pipelines using ultrasonic modification and heat shrinkage. Part 1. Aspects of connection and restoration of polymeric pipelines for gas transport. Chem. and Petrol. Eng. 47, 204-209.http://dx.doi.org/10.1007/s10556-011-9447-5.

[29] Kolosov AE, Sakharov OS, Sivetskii VI, Sidorov DE \& Pristailov SO (2011), Effective hardware for connection and repair of polyethylene pipelines using ultrasonic modification and heat shrinkage. Part 2. Production bases for molding of epoxy repair couplings with shape memory. Chem. and Petrol. Eng. 47, 210.http://dx.doi.org/10.1007/s10556-011-9448-4.

[30] Kolosov AE, Sakharov OS, Sivetskii VI, Sidorov DE \& Pristailov SO (2011), Effective hardware for connection and repair of polyethylene pipelines using ultrasonic modification and heat shrinkage. Part 3. Analysis of surface-treatment methods for polyethylene pipes connected by banding. Chem. and Petrol. Eng. 47, 216.http://dx.doi.org/10.1007/s10556-011-9449-3.

[31] Kolosov AE, Sakharov OS, Sivetskii VI, Sidorov DE \& Pristailov SO (2011), Effective hardware for connection and repair of polyethylene pipelines using ultrasonic modification and heat 
shrinkage. Part 4. Characteristics of practical implementation of production bases developed using epoxy-glue compositions and banding. Chem. and Petrol. Eng. 47, 280. http://dx.doi.org/10.1007/s10556-011-9460-8.

[32] Kolosov AE, Sakharov OS, Sivetskii VI, Sidorov DE \& Pristailov SO (2011), Effective hardware for connection and repair of polyethylene pipelines using ultrasound modification and hea shrinking. Part 5. Aspects of thermistor couplings and components used in gas-pipeline repair. Chem. and Petrol. Eng. 47285. http://dx.doi.org/10.1007/s10556-011-9461-7.

[33] Kolosov AE (2015), Preparation of Nano-Modified Reactoplast Polymer Composites. Part 1. Features of used nanotechnologies and potential alication areas of nanocomposites (a review). Chem. and Petrol. Eng. 51, I. 7-8, 569-573. http://dx.doi.org/10.1007/s10556015-0088-y.

[34] Kolosov AE (2016), Preparation of Reactoplastic Nanomodified Polymer Composites. Part 2. Analysis of means of forming nanocomposites (patent review). Chem. and Petrol. Eng. 51, I. 9 10, 640-645. http://dx.doi.org/10.1007/s10556-016-0100-1.

[35] Kolosov AE (2016), Preparation of Reactoplastic Nanomodified Polymer Composites. Part 3. Methods for dispersing carbon nanotubes in organic solvents and liquid polymeric media (review). Chem. and Petrol. Eng. 52, I. 1-2, 7176.http://dx.doi.org/10.1007/s10556-016-0151-3.

[36] Kolosov AE (2016), Preparation of Reactoplastic Nanomodified Polymer Composites. Part 4. Effectiveness of modifying epoxide oligomers with carbon nanotubes (review). Chem. and Petrol. Eng. 52, I. 7-8, 573-577. http://dx.doi.org/10.1007/s10556-016-0235-0.

[37] Kolosov AE (2017), Preparation of Reactoplastic Nano-Modified Polymer Composites. Part 5. Advantages of using nano-modified structural carbon-fiber composites (a review). Chem. and Petrol. Eng. 52, I. 9-10, 721-725. http://dx.doi.org/10.1007/s10556-0170259-0.

[38] available online: https://www.hielscher.com/ru/ultrasonicgraphene-preparation.htm, last visit:10.10.2013

[39] An X, Simmons, T, Shah, R, Wolfe C, Lewis KM, Washington M, Nayak, SK, Talapatra, S, Kar, S (2010), Stable Aqueous Dispersions of Noncovalently Functionalized Graphene from Graphite and their Multifunctional High-Performance Applications. Nano Letters 10, 4295-4301.

[40] Bang JH, Suslick KS (2010), Applications of Ultrasound to the Synthesis of Nanostructured Materials. Advanced Materials 22, 1039-1059.

[41] Kolosov AE \& Kolosova EP, Functional Materials for Construction Application Based on Classical and Nano Composites: Production and Properties. In: Rita Khanna, Romina Cayumil (Eds.), Recent Developments in the field of Carbon Fibers, InTechOpen, (2018), ISBN: 978-953-51-6055-7. 